\title{
THE ROLE OF TITANIUM MESH IN LIMITING DIMENSIONAL CHANGES OF MAXILLARY SINUS GRAFT: RANDOMIZED CLINICAL TRIAL
}

\begin{abstract}
Objectives: The dimensional changes that occurs during the healing of the grafting material in external sinus lift procedure is always present and sometimes may lead to difficulties in placing the dental implants in the second stage. This study aims to evaluate the efficacy of adding a titanium mesh as a new fixed floor of the sinus to minimize these changes.
\end{abstract}

Materials and Methods: this randomized clinical trial included 8 patients who needed bilateral 2-stages sinus lift. On one side sinus lift was carried out using Deproteinized Bovine Bone Matrix (DBBM) mixed with A-PRF+ (Group A) while on the other side a titanium mesh was fixed as a new sinus floor above the same mixture (Group B). CBCT radiographs were taken preoperatively (T0), directly postoperatively (T1), and 9 months after surgery (T2). Linear measurements were recorded in the same points in all three radiographs, graft volume was measured and compared using 3D Slicer software.

Results: the mean bone height $(\mathrm{BH})$ at $\mathrm{T} 1$ was 13.08 and $13.56 \mathrm{~mm}$ while at $\mathrm{T} 2$ was 11.56 and $13.56 \mathrm{~mm}$ in groups $\mathrm{A}$ and $\mathrm{B}$ respectively. The mean reduction in $\mathrm{BH}$ was 1.68 and $0.00 \mathrm{~mm}$ in groups $\mathrm{A}$ and $\mathrm{B}$ respectively. The graft volume (GV) at $\mathrm{T} 1$ was 1.49 and $1.63 \mathrm{cc}$ and at $\mathrm{T} 2$ was 1.19 and $1.53 \mathrm{cc}$, the mean volume shrinkage was 0.29 and 0.1 cc in groups A and B respectively. The differences between the groups in both linear and volumetric changes were statistically significant.

Conclusions: within the limitation of this study it may be concluded that adding a titanium mesh as a new floor of the sinus helps limiting the shrinkage of both the linear dimensions and overall graft volume.

Key Words: Cone-beam computed tomography, deproteinized bovine bone matrix, lateral sinus lift, sinus augmentation, titanium mesh.
*Sammy J. Fattouh ${ }^{1}$

Suleiman T. Dayoub ${ }^{1}$

Ahmad I. AL-Manadili²

ORCID IDs of the authors:

S.J.F. $\quad 0000-0002-1818-9759$

S.T.D. $\quad 0000-0003-0671-9473$

A.I.A.M. 0000-0003-0351-8609
${ }^{1}$ Department of Periodontology, Faculty of Dentistry, Damascus University, Damascus, Syria.

${ }^{2}$ Department of Oral Pathology, Faculty of Dentistry, Damascus University, Damascus, Syria.
Received : 23.06 .2020

Accepted : $: 21.10 .2020$ 


\section{INTRODUCTION}

Placing dental implants in posterior Maxilla might be inapplicable due to sinus pneumatization and thus require sinus lift procedure to augment the bone in this area prior to implant placement.

Sinus lift technique was introduced by Tatum $e t$ $a l .{ }^{1}$ where he proposed creating a lateral window to gain access to the Schneiderian membrane and elevate it. Boyne and James ${ }^{2}$ introduced the use of autogenous bone to Tatum original procedure but later several biomaterials were introduced to the technique including Deproteinized Bovine Bone Matrix (DBBM), Alloplasts, Allografts and even Platelet rich fibrin (PRF). ${ }^{3,4}$ A healing time of 6-9 months is required depending on the biomaterial used.

During that time a dimensional change to the graft occur due to continuous pneumatization of the sinus and remodeling of the graft particles and the collapse of the gaps between these particles. ${ }^{5}$ The percentage of the shrinkage both linear and volumetric varies depending on the biomaterial used. Zhang et al. ${ }^{6}$ found a $16 \%$ linear and $22 \%$ volume shrinkage of DBBM. Another study reported $10 \%$ shrinkage of DBBM after 8-9 months $^{7}$, while this percentage rises to $17.65 \%$ when using calcium phosphate with DBBM mixture in 2:1 ratio $^{8}$, and $13 \%$ in hydroxyapatite and autogenous bone mixture. ${ }^{9}$ Allografts seems to undergo more resorption as only $82 \%$ of allografts remained after 6 months and $60 \%$ after one year. ${ }^{10}$ In a systematic review about dimensional changes after lateral sinus lift Shanbhag et al. ${ }^{11}$ reported that graft resorption is inevitable especially with autogenous bone which reach $45 \%$ while when other biomaterials or the mixture of autogenous bone with biomaterials are used this percentages varies between $18-22 \%$ although this reduction in graft volume didn't seem to effect implant placement or survival.

Cone-Beam Computed Tomography (CBCT) is a reliable diagnostic tool and is widely used in the treatment planning of dental implants and in the diagnosis of several pathologies in the jaws. ${ }^{12}$ CBCT was successfully used to follow the changes in graft dimension in several studies by using many computer softwares..$^{6-11,13-16}$
Choukroun et al. ${ }^{17}$ first introduced the concept of Platelet Rich Fibrin (PRF) and was under constant development so the same author introduced Advanced PRF Plus (A-PRF+) which is a more advanced form of PRF based on low centrifugation speed and less time thus trapping a higher number of leukocytes in the fibrin matrix and therefore increasing cytokines release from it.

PRF became widely used in regenerative dentistry because of its ability to promote angiogenesis and growth factors release ${ }^{18}$, PRF was used in improving the healing of extraction sites ${ }^{19}$, with immediate implant placement ${ }^{20}$, in the treatment of Periimplantitis ${ }^{21}$, in mucogingival surgeries ${ }^{22,23}$, and in the treatment of periodontitis. ${ }^{24-28}$

PRF was used in sinus lifting in several studies either alone or in a mixture with DBBM. The benefit of this mixture was advocated by the authors who reported better new bone formation and faster healing ${ }^{29,30}$, however this subject is still controversial as a recent meta-analysis showed no evidence on the necessity of adding PRF to the grafting material. ${ }^{31}$

In a case series, Atef et al. ${ }^{32}$ used a titanium mesh as space maintainer in a lateral graftless sinus lift and found promising results so Bahaa-eldin et $a l .{ }^{33}$ performed a pilot study where they were able to place an implant in the newly formed bone under the titanium mesh but they reported that the density of this bone was far less than that of the bovine xenograft.

We proposed a modification to Atef et al. ${ }^{32}$ technique by placing a titanium mesh on top of the graft material as a new floor of the elevated sinus and fixing this mesh with titanium screws to the lateral wall of the sinus to minimize the dimensional change of the graft material.

The aim of this study was to evaluate the dimensional changes in a grafted sinus with xenograft mixed with A-PRF+ compared to adding a titanium mesh on top of the same mixture as a new floor of the elevated sinus. 


\section{MATERIAL AND METHODS}

\section{Study Design}

This research was approved by Damascus University ethical committee for scientific research ID\#1926 dated 14/5/2018 and the study protocol adhere to the international agreements (Helsinki Declaration revised 2008)

The study is a split mouth design clinical trial where a standard sinus lift is performed on one side using DBBM mixed with A-PRF+ (Control Group - Group A) and on the other side the same mix was used in addition to a titanium mesh (Test Group Group B).

\section{Patient's recruitment}

8 patients who visited Damascus Universityfaculty of dentistry- Department of Periodontology - post-graduate clinic and needed bilateral sinus lift procedure were invited to participate in the study.

Inclusion criteria included 6 months since the last extraction, no smoking, residual bone height less than $5 \mathrm{~mm}$ while exclusion criteria included pregnancy, diabetes, current treatment with corticosteroids and smoking.

After explaining the procedure, a written consent was signed by each participant. The sides were allocated randomly using a coin toss.

\section{Surgical Procedure}

The sinus lift procedure was carried out following the protocol proposed by Tatum et al. ${ }^{1}$, after flap elevation and the exposure of the lateral sinus wall a bony window was prepared using piezoelectric instrument, then the Schneiderian membrane was elevated by manual instruments. The bony window was left in place and elevated with the membrane.

After the completion of the elevation A-PRF+ was prepared following the original protocol by Choukroun ${ }^{17}$ and then mixed with the DBBM biomaterial (Bonefill ${ }^{\circledR}$ Mix, Bionnovation, Bauru, Sao Paolo, Brazil) and the mix is used to fill the sinus and finally the osteotomy was covered by a collagen membrane (Biocollagen ${ }^{\circledR}$, Bioteck S.p.A., Arcugnano, Vicenza, Italy).

In the test group the depth of the sinus was measured using a periodontal probe, then a piece of titanium mesh (Titanium Wire Mesh, Orthomax,
Vadodara, Gujarat, India) was cropped to the desired dimensions and fixed on the lateral wall of the sinus using $4 \mathrm{~mm}$ titanium screw (Mini Screw $\varnothing 1.5 \mathrm{~mm}$, Orthomax, Vadodara, Gujarat, India) (Figure 1B).

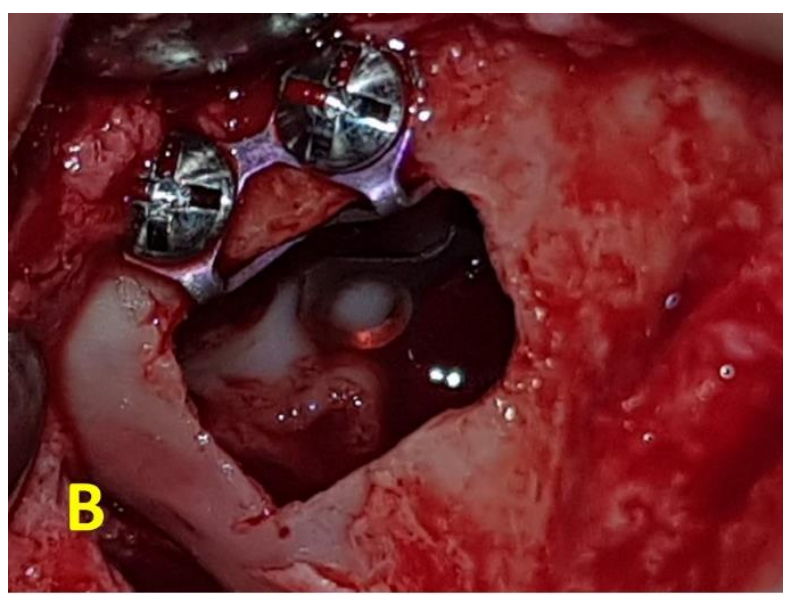

Figure 1B. The titanium mesh is fixed on the lateral wall of the sinus with titanium screws

Another modification was introduced to the original Atef $\mathrm{et} \mathrm{al.}{ }^{32}$ technique where they cropped the mesh in an (L) shape while we cropped and then bend the mesh in a $\left(\mathrm{V}^{-}\right)$shape (Figure 1A) which also provided additional $2-3 \mathrm{~mm}$ lift above the upper margin of the bony window.

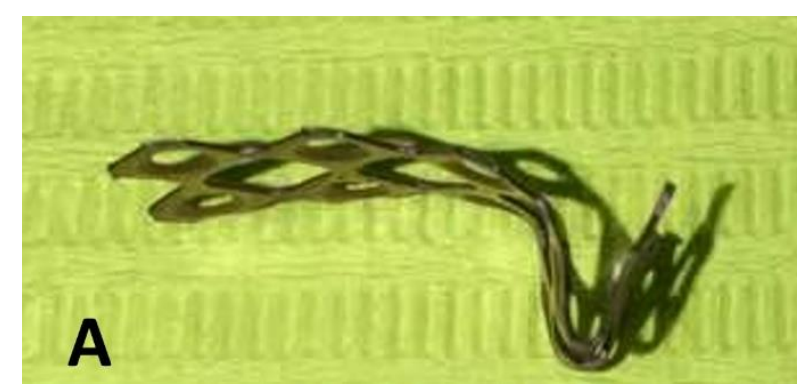

Figure 1A. the bended titanium mesh,

We also used some A-PRF+ membranes as pads under the mesh to avoid any tear to the membrane while placing the mesh.

In case a membrane perforation occurred A$\mathrm{PRF}+$ membranes were used along with a piece of collagen membrane to cover the perforation.

Patients were informed that this mesh will not be removed in the future as it will be incorporated in the newly formed bone and in close contact with the Schneiderian membrane which will make its removal impossible without damaging the membrane. 


\section{Radiographic assessment}

A cone beam CT radiograph was taken before (T0) and immediately after the operation (T2) and after 9 months (T3) (Figure 2).

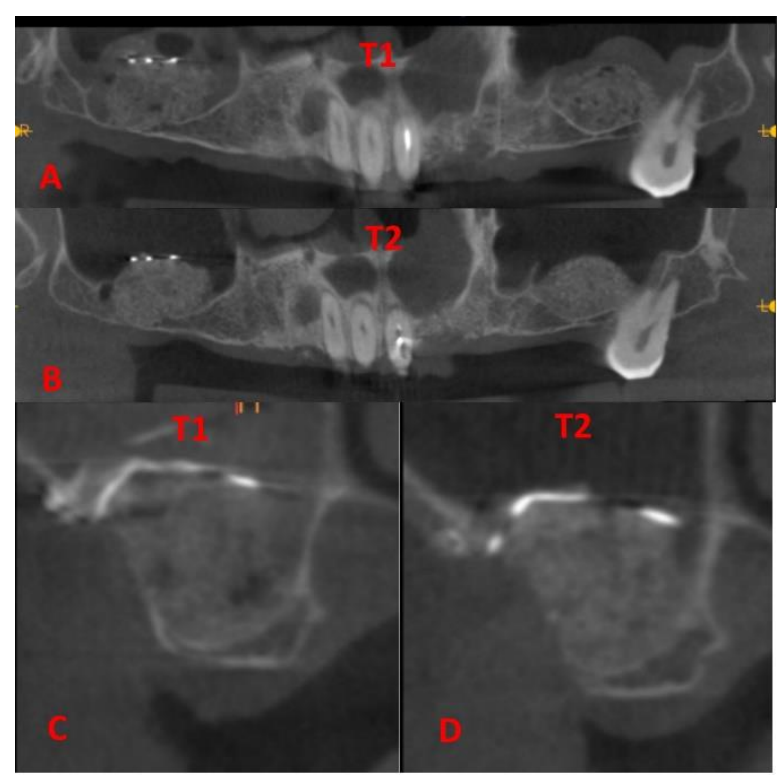

Figure 2: A) Panoramic reformatting of a patient directly after surgery, B) Panoramic reformatting of the same patient after 9 months, C) Oblique slice in the middle of the titanium mesh directly after surgery, D) Oblique slice in the same point after 9 months

Linear radiographic evaluation was carried out by taking measurements in the same points and directions in all three CBCT radiographs. These points were determined depending on fixed anatomical landmark to match all three radiographs like neighboring teeth and nasal septum based on the technique described by Anduze-Acher et al. ${ }^{34}$ The distance between the nasal septum and the slice of interest was measured and applied in all three radiographs while the direction of measurement was determined by repeating an angel between the direction of measurement and a horizontal line tangent to the lowest point of the alveolar crest.

$3 \mathrm{~d}$ volumetric measurements were recorded using 3D Slicer software were the segment editor tool was used to mark the graft boundaries in all slices then the software calculates the volume using the segment statistics tool. ${ }^{35}$

\section{Statistical Analysis}

Data were analyzed using statistical software (IBM SPSS Statistics version 22). Unpaired Student tTest was used to analyze the difference in bone dimensions during healing process between the two groups. Results were considered significant at $\mathrm{P}<.05$ with $95 \%$ confidence intervals.

\section{RESULTS}

This study included 8 patients (5women, 3men) with mean age (52.13 years) who needed bilateral sinus lifting without implant placement.

The differences in mean bone height $(\mathrm{BH})$ (Table 1) before the surgery and the graft height directly after surgery was not statistically significant between the two groups. While it was higher in the test group after 9 months with statistically significant results.

Table 1: Mean Bone Hight Value in the study groups compared before (T0), directly after surgery (T1) and after 9 months (T2)

\begin{tabular}{lllllll}
\hline & T0 & & T1 & \multicolumn{2}{c}{ T2 } \\
\cline { 2 - 7 } & BH & $p$ value & BH & $p$ value & BH & $p$ value \\
Group A & $2.41 \pm 1.06$ & 0.613 & $13.08 \pm 1.21$ & 0.389 & $11.39 \pm 1.65$ & $0.006^{*}$ \\
\hline
\end{tabular}

BH: Bone Height (mm)

$*: \mathrm{P}<0.05$ is considered statistically significant

The mean graft height (GH) (Table 2) directly after surgery was not statistically significant between the groups but after 9 months the mean graft height was $8.99 \pm 1.93$ in group $A$ and $10.88 \pm 0.58$ in Group $\mathrm{B}$ and this difference between the groups was statistically significant.

Table 2: Mean Graft Hight and 2d Shrinkage compared between the study groups directly after surgery (T1) and after 9 months (T2).

\begin{tabular}{lllllll}
\hline & T1 & & T2 & & Graft Shrinkage \\
\cline { 2 - 7 } & GH & $p$ value & GH & $p$ value & 2D & $p$ value \\
Group A & $10.67 \pm 0.94$ & 0.604 & $8.99 \pm 1.93$ & & $1.68 \pm 1.21$ & 0.019 \\
& $10.88 \pm 0.58$ & & $10.88 \pm 0.58$ & & $(15.74 \%)$ & $0.001 *$ \\
\hline
\end{tabular}

GH: Graft Height (mm)

$*: \mathrm{P}<0.05$ is considered statistically significant 
The mean linear bone height reduction in the control group was $1.68 \mathrm{~mm}$ while in the test group when the measurements were made directly under the titanium mesh there was no reduction in bone height and these differences were statistically significant (Table 2).
The mean graft volume (GV) directly after surgery was not statically different between the two groups. At 9 months after surgery graft volume was significantly higher in the test group and the mean graft volume shrinkage was 0.29 and $0.10 \mathrm{cc}$ in group A and group B respectively and there was a statistically significant difference between the groups. (Table 3)

Table 3: Mean Graft Volume and 3d Shrinkage compared between the study groups directly after surgery (T1) and after 9 months(T2).

\begin{tabular}{lllllll}
\hline & T1 & & T2 & \multicolumn{3}{c}{ Graft Shrinkage } \\
\cline { 2 - 7 } & GV & $p$ value & GV & $p$ value & 3D & $p$ value \\
Group A & $1.49 \pm 0.35$ & & $1.19 \pm 0.30$ & & $0.29 \pm 0.11$ & \\
& & 0.398 & & 0.039 & $\begin{array}{l}(20.01 \%) \\
0.10 \pm 0.05\end{array}$ & $0.000^{*}$ \\
Group B & $1.63 \pm 0.31$ & & $1.53 \pm 0.29$ & & $(5.93 \%)$ & \\
\hline
\end{tabular}

GV: Graft Volume (cc)

*: $\mathrm{P}<0.05$ is considered statistically significant

\section{DISCUSSION}

As far to the author's knowledge this is the first split mouth randomized clinical trial that studies the use of titanium mesh as a space maintainer in two stages sinus lift surgery using DBBM mixed with A-PRF+ as grafting material.

The shrinkage of grafting material, both linear and volumetric is an inevitable outcome of the healing process of sinus grafts. The clinicians are advised to increase the amount of biomaterials introduced to the sinus to compensate for this remodeling process. ${ }^{11}$

In the present study we found $1.68 \mathrm{~mm}$ $(15.74 \%)$ linear shrinkage of the graft material in the control group between follow-ups, this reduction came in accordance with other similar studies in the literature that reported variable amount of shrinkage of the grafting material. ${ }^{6,36-38}$

This shrinkage is mainly due the continuous pneumatization of the sinus and the pressure it implicates on the grafting material during breathing and accelerate resorption of the grafting material especially in two stage sinus lift this process is also influenced by the properties of the biomaterial used. ${ }^{8}$ Multiple tooth lost, the angel between the medial and lateral sinus wall, and large sinus may be associated with higher graft contraction. ${ }^{6}$
In the study group the installment of the titanium mesh as a new floor of the elevated sinus provided protection for the graft material from the continuous pressure thus allowed the biomaterial to be remodeled and replaced by new host bone without loss of height. This space maintenance effect of the mesh is one of the fundamental principles of guided bone regeneration ${ }^{39}$, as this protected space is essential for bone graft healing by hard tissue cells which will regenerate the bone during the healing and maturation time. ${ }^{40}$ The comparison between the two groups showed statistically significant difference in term of linear measurement reduction over time.

Volumetric analysis of the graft showed some shrinkage in both groups and this shrinkage was statistically significant between the two groups. In the control group the mean volume shrinkage was $0.29 \mathrm{cc}(20 \%)$ which corelate to the previously established graft shrinkage in the literature. . $^{6}$ $8,10,11,16,36$ In the test group the applied mesh covered most of the graft but on the sides the graft was still subject to some pressure thus explaining the minor reduction in volume with an average of $0.1 \mathrm{cc}$ (5.93\%). As a study by Guo et al. ${ }^{41}$ showed an overall reduction of DBBM graft volume by $19.4 \%$ and more specifically $0.29 \%$ in the bucco-palatal, $5.87 \%$ in the mesio-distal, and $14.32 \%$ in the vertical direction thus explaining the finding of our study where the titanium mesh offered protection 
to the graft from the shrinkage in the vertical dimension while it was still under some pressure in the mesio-distal direction which resulted in the statically significant less mean graft volume reduction in the test group compared to the control group.

This study had some limitations, such as the width of the titanium mesh was limited to $10 \mathrm{~mm}$ or $15 \mathrm{~mm}$ due to its design so in all the cases the mesh's width was $10 \mathrm{~mm}$ and sometimes even if we had a wider osteotomy/Sinus we were not able to place wider mesh and as mentioned before the effect of the mesh application was at its best directly under the mesh so for future study we recommend using a more flexible mesh design that allows to perfectly crop the mesh to the width of the osteotomy.

We also believe that the real benefit of the protection offered by the mesh application is when using other than the bovine biomaterial e.g.: Allograft/autogenous alone bone or mixed with DBBM which are more prone to resorb during the healing time thus we recommend to study the application of the titanium mesh above these biomaterials.

\section{CONCLUSIONS}

Within the limitation of this study it may be concluded that adding a titanium mesh as a new floor of the elevated sinus helped in protecting the underling graft from forces applied from continuous pneumatization thus eliminating linear shrinkage directly under the mesh and minimizing the overall shrinkage of the graft.

This technique may be applied to improve the final outcome of sinus lift procedure and make it more predictable and guarantee a sufficient graft volume after healing time thus facilitate implant placement.

\section{ACKNOWLEDGMENTS}

The authors claim to have no financial interest, either directly or indirectly, in the products or information listed in the article.

\section{REFERENCES}

1. Tatum OH, Lebowitz MS, Tatum CA, Borgner RA. Sinus augmentation. Rationale, development, long-term results. N Y State Dent J 1993;59:43-48.

2. Boyne PJ, James R. Grafting of the maxillary sinus floor with autogenous marrow and bone. J Oral Surg 1980;38:613-616.

3. Danesh-Sani SA, Engebretson SP, Janal MN. Histomorphometric results of different grafting materials and effect of healing time on bone maturation after sinus floor augmentation: a systematic review and meta-analysis. J Periodontal Res 2017;52:301-312.

4. Ali S, Bakry SA, Abd-Elhakam H. Platelet-Rich Fibrin in Maxillary Sinus Augmentation: A Systematic Review. J Oral Implantol 2015;41:746-753.

5. Ahmet S, Gultekin BA, Karabuda ZC, Olgac V. Two composite bone graft substitutes for maxillary sinus floor augmentation: Histological, histomorphometric, and radiographic analyses. Implant Dent 2016;25:313321.

6. Zhang L, Si M, Shi J, Yang G, Shi Y. Evaluation of three-dimensional contraction of the volume of grafts after staged augmentation of the sinus floor, and an analysis of influential factors. Br J Oral Maxillofac Surg 2019;57:323-329.

7. Mazzocco F, Lops D, Gobbato L, Lolato A, Romeo

E, Del Fabbro M. Three-Dimensional Volume Change of Grafted Bone in the Maxillary Sinus. Int J Oral Maxillofac Implants 2014;29:178-184.

8. Gultekin BA, Borahan O, Sirali A, Karabuda ZC, Mijiritsky E. Three-Dimensional Assessment of Volumetric Changes in Sinuses Augmented with Two Different Bone Substitutes. Biomed Res Int 2016;2016:1-7.

9. Wanschitz F, Figl M, Wagner A, Rolf E. Measurement of volume changes after sinus floor augmentation with a phycogenic hydroxyapatite. Int J Oral Maxillofac Implants 2006;21:433-438.

10. Kim E-S, Moon S-Y, Kim S-G, Park H-C, Oh J-S. Three-Dimensional Volumetric Analysis After Sinus Grafts. Implant Dent 2013;22:170-174.

11. Shanbhag S, Shanbhag V, Stavropoulos A. Volume Changes of Maxillary Sinus Augmentations over Time: A Systematic Review. Int J Oral Maxillofac Implants 2014;29:881-892.

12. Macleod I, Heath N. Cone-Beam Computed Tomography (CBCT) in Dental Practice. Dent Update 2008;35:590-598.

13. Berberi A, Bouserhal L, Nader N, et al. Evaluation of Three-Dimensional Volumetric Changes After Sinus Floor Augmentation with Mineralized Cortical Bone 
Allograft. J Maxillofac Oral Surg. 2015;14:624-629.

14. Temmerman A, Van Dessel J, Cortellini S, Jacobs R, Teughels W, Quirynen M. Volumetric changes of grafted volumes and the Schneiderian membrane after transcrestal and lateral sinus floor elevation procedures: A clinical, pilot study. J Clin Periodontol 2017;44:660671.

15. Arasawa M, Oda Y, Kobayashi T, et al. Evaluation of bone volume changes after sinus floor augmentation with autogenous bone grafts. Int J Oral Maxillofac Surg 2012;41:853-857.

16. Younes F, Cosyn J, De Bruyckere T, Cleymaet R, Eghbali A. A 2-year prospective case series on volumetric changes, PROMs, and clinical outcomes following sinus floor elevation using deproteinized bovine bone mineral as filling material. Clin Implant Dent Relat Res 2019;2:301-309.

17. Miron RJ, Aalam AA, Fujioka-Kobayashi M, Guillemette V. Autogenous Blood-Derived Barrier Membranes: Platelet-Rich Fibrin in Regenerative Dentistry. In: Miron RJ, Zhang Y (eds). NextGeneration Biomaterials for Bone and Periodontal Regenration. Batavia: Quintessence Publishing Company Incorporated; 2019:169-184.

18. Miron RJ, Pikos MA, Wang H-L. Maxillary Sinus Floor Elevation Procedures with Platelet Rich Fibrin: Indications and Clinical Recommendations. In: Miron RJ, Choukroun J (eds). Platelet Rich Fibrin in Regenerative Dentistry: Biological Background and Clinical Indications.Hoboken: John Wiley \& Sons, Ltd; 2017:99-113.

19. Ercan E. The effect of different platelet-rich concentrates on extraction site healing. Cumhur Dent $\mathbf{J}$ 2018;21:304-310.

20. Boora P. Effect of Platelet Rich Fibrin (PRF) on Peri-implant Soft Tissue and Crestal Bone in One-Stage Implant Placement: A Randomized Controlled Trial. J Clin Diagnostic Res 2015;9:18-21.

21. Hamzacebi B, Oduncuoglu B, Alaaddinoglu E. Treatment of Peri-implant Bone Defects with PlateletRich Fibrin. Int $\mathrm{J}$ Periodontics Restor Dent 2015;35:415-422.

22. Sreedhar A, Padma R, Shilpa A, Kumar P, Nagasri M, Kumar C. A split mouth randomized controlled study to evaluate the adjunctive effect of platelet-rich fibrin to coronally advanced flap in Miller's class-I and II recession defects. J Indian Soc Periodontol 2013;17:631-636.
23. Tunalı M, Özdemir H, T A, B G, L P, E. F. Clinical evaluation of autologous platelet-rich fibrin in the treatment of multiple adjacent gingival recession defects: a 12-month study. - PubMed - NCBI. Int J Periodontics Restor Dent 2015;35:105-114.

24. Shah M, Patel J, Dave D, Shah S. Comparative evaluation of platelet-rich fibrin with demineralized freeze-dried bone allograft in periodontal infrabony defects: A randomized controlled clinical study. J Indian Soc Periodontol 2015;19:56-60.

25. Bains V, Gupta V, Jhingran R, Mathur A, Singh G. Evaluation of intrabony defects treated with platelet-rich fibrin or autogenous bone graft: A comparative analysis. Eur J Dent 2015;9:100-108.

26. Ajwani H, Shetty S, Dharmarajan G, Rahul K. Comparative Evaluation of Platelet-Rich Fibrin Biomaterial and Open Flap Debridement in the Treatment of Two and Three Wall Intrabony Defects. J Int Oral Heal 2015;7:32-37.

27. Shah M, Deshpande N, Bharwani A, Nadig P, Doshi $\mathrm{V}$, Dave D. Effectiveness of autologous platelet-rich fibrin in the treatment of intra-bony defects: A systematic review and meta-analysis. J Indian Soc Periodontol 2014;18:698-704.

28. Rosamma Joseph V, Sam G, Vijay Amol N. Clinical evaluation of autologous platelet rich fibrin in horizontal alveolar bony defects. J Clin Diagnostic Res 2014;8:ZC43-ZC47.

29. Tanaka H, Toyoshima T, Atsuta I, et al. Additional effects of platelet-rich fibrin on bone regeneration in sinus augmentation with deproteinized bovine bone mineral: Preliminary results. Implant Dent 2015;24:669674.

30. Pichotano EC, de Molon RS, de Souza RV, Austin RS, Marcantonio E, Zandim-Barcelos DL. Evaluation of L-PRF combined with deproteinized bovine bone mineral for early implant placement after maxillary sinus augmentation: A randomized clinical trial. Clin Implant Dent Relat Res 2019;21:253-262.

31. Liu R, Yan M, Chen S, Huang W, Wu D, Chen J. Effectiveness of Platelet-Rich Fibrin as an Adjunctive Material to Bone Graft in Maxillary Sinus Augmentation: A Meta-Analysis of Randomized Controlled Trails. Biomed Res Int 2019;2019:1-10.

32. Atef M, Hakam MM, ElFaramawey MI, AbouElFetouh A, Ekram M. Nongrafted Sinus Floor Elevation with a Space-Maintaining Titanium Mesh: Case-Series Study on Four Patients. Clin Implant Dent 
Relat Res 2014;16:893-903.

33. Bahaa-eldin K, Mostafa B, Nasry S, Reda A, Shoeib

M. Maxillary Sinus Augmentation Using a Titanium Mesh : A Randomized Clinical Trial 2017;5:359-369.

34. Anduze-Acher G, Brochery B, Felizardo R, Valentini P, Katsahian S, Bouchard P. Change in sinus membrane dimension following sinus floor elevation: A retrospective cohort study. Clin Oral Implants Res 2013;24:1123-1129.

35. Kikinis R, Pieper SD, Vosburgh KG. Intraoperative Imaging and Image-Guided Therapy. Intraoperative Imaging Image-Guided Ther. Published online 2014:277-289.

36. Umanjec-Korac S, Wu G, Hassan B, Liu Y, Wismeijer D. A retrospective analysis of the resorption rate of deproteinized bovine bone as maxillary sinus graft material on cone beam computed tomography. Clin Oral Implants Res 2014;25:781-785.

37. Yin L, Yu Z, Chen Z, et al. Analysis of Bone Height Changes after Maxillary Sinus Augmentation with
Simultaneous and Delayed Placement of Dental Implants: A Clinical and Radiographic Study. J Prosthodont 2016;25:440-445.

38. Hieu PD, Chung JH, Yim S Bin, Hong KS. A radiographical study on the changes in height of grafting materials after sinus lift: A comparison between two types of xenogenic materials. J Periodontal Implant Sci 2010;40:25-32.

39. Wang HL, Boyapati L. "pASS" principles for predictable bone regeneration. Implant Dent. 2006;15:817.

40. Gultekin BA, Cansiz E, Yalcin S. Ridge Augmentation Techniques in Preprosthetic Implant Surgery. In: Motamedi MH (ed). A Textbook of Advanced Oral and Maxillofacial Surgery Volume 3. London:InTech; 2016:245-268.

41. Guo X, Jiang Q, Ruan H, Luo Y, Yu Y. [Evaluation of three-dimensional changes after sinus floor augmentation with DBBM]. Shanghai Kou Qiang Yi Xue 2013;22:448-452. 